\title{
WASP-104b and WASP-106b: two transiting hot Jupiters in 1.75-day and 9.3-day orbits ${ }^{\star} \star \star \star$
}

\author{
A. M. S. Smith ${ }^{1,2}$, D. R. Anderson ${ }^{2}$, D. J. Armstrong ${ }^{3}$, S. C. C. Barros ${ }^{4}$, A. S. Bonomo ${ }^{5}$, F. Bouchy ${ }^{4}$, D. J. A. Brown ${ }^{3}$, \\ A. Collier Cameron ${ }^{6}$, L. Delrez ${ }^{7}$, F. Faedi ${ }^{3}$, M. Gillon ${ }^{7}$, Y. Gómez Maqueo Chew ${ }^{3}$, G. Hébrard ${ }^{8,9}$, E. Jehin ${ }^{7}$, \\ M. Lendl ${ }^{7,10}$, T. M. Louden ${ }^{3}$, P. F. L. Maxted ${ }^{2}$, G. Montagnier ${ }^{8,9}$, M. Neveu-VanMalle ${ }^{10,11}$, H. P. Osborn ${ }^{3}$, F. Pepe ${ }^{10}$, \\ D. Pollacco ${ }^{3}$, D. Queloz ${ }^{10,11}$, J. W. Rostron ${ }^{3}$, D. Segransan ${ }^{10}$, B. Smalley ${ }^{2}$, A. H. M. J. Triaud ${ }^{10,12}$, O. D. Turner ${ }^{2}$, \\ S. Udry ${ }^{10}$, S. R. Walker ${ }^{3}$, R. G. West ${ }^{3}$, and P. J. Wheatley ${ }^{3}$
}

${ }^{1}$ N. Copernicus Astronomical Centre, Polish Academy of Sciences, Bartycka 18, 00-716 Warsaw, Poland e-mail: amss@camk.edu.pl

2 Astrophysics Group, Lennard-Jones Laboratories, Keele University, Keele, Staffordshire, ST5 5BG, UK

3 Department of Physics, University of Warwick, Coventry CV4 7AL, UK

4 Aix Marseille Université, CNRS, LAM (Laboratoire d'Astrophysique de Marseille) UMR 7326, 13388 Marseille, France

5 INAF - Osservatorio Astrofisico di Torino, via Osservatorio 20, 10025 Pino Torinese, Italy

6 SUPA, School of Physics and Astronomy, University of St. Andrews, North Haugh, St. Andrews, Fife, KY16 9SS, UK

7 Institut d'Astrophysique et de Géophysique, Université de Liège, Allée du 6 Août 17, Sart Tilman, Liège 1, Belgium

8 Institut d'Astrophysique de Paris, UMR7095 CNRS, Université Pierre \& Marie Curie, 98bis boulevard Arago, 75014 Paris, France

9 Observatoire de Haute-Provence, CNRS/OAMP, 04870 Saint-Michel-1'Observatoire, France

10 Observatoire de Genève, Université de Genève, 51 Chemin des Maillettes, 1290 Sauverny, Switzerland

11 Cavendish Laboratory, J J Thomson Avenue, Cambridge CB3 0HE, UK

12 Kavli Institute for Astrophysics \& Space Research, Massachusetts Institute of Technology, Cambridge, MA 02139, USA

Received 5 August 2014 / Accepted 9 September 2014

\section{ABSTRACT}

\begin{abstract}
We have used the WASP survey to discover two exoplanetary systems, each consisting of a Jupiter-sized planet transiting an 11th-magnitude $(V)$ main-sequence star. WASP-104b orbits its star in $1.75 \mathrm{~d}$, whereas WASP-106b has the fourth-longest orbital period of any planet discovered by means of transits observed from the ground, orbiting every $9.29 \mathrm{~d}$. Each planet is more massive than Jupiter (WASP-104b has a mass of $1.27 \pm 0.05 M_{\text {Jup }}$, while WASP-106b has a mass of $1.93 \pm 0.08 M_{\text {Jup }}$ ). Both planets are just slightly larger than Jupiter, with radii of $1.14 \pm 0.04$ and $1.09 \pm 0.04 R_{\text {Jup }}$ for WASP-104 and WASP-106, respectively. No significant orbital eccentricity is detected in either system, and while this is not surprising in the case of the short-period WASP-104b, it is interesting in the case of WASP-106b, because many otherwise similar planets are known to have eccentric orbits.
\end{abstract}

Key words. planets and satellites: detection - planets and satellites: fundamental parameters - stars: individual: WASP-104b stars: individual: WASP-106b - planetary systems

\section{Introduction}

Transiting planets are vital for our understanding of planetary systems, because their radii and absolute masses can be measured. Recently, the large number of planets and planetary candidates discovered by the Kepler satellite has extended the parameter space of transiting planet discovery and led to major advances in our understanding of the statistics of the Galactic planetary population. Planets transiting stars brighter than those observed by Kepler are required to increase our knowledge of the range of properties exhibited by the nearby planetary population, as well as to make advances in our understanding of planetary formation and evolution. Examples of planet characterisation observations that require bright target stars include high-precision radial velocity (RV) measurements to determine orbital obliquities and measurements of planetary transmission and emission spectra to

\footnotetext{
* Table 1 is available in electronic form at http://www . aanda.org $\star \star$ The table of photometry is only available at the CDS via anonymous ftp to cdsarc.u-strasbg. fr (130.79.128.5) or via http://cdsarc.u-strasbg.fr/viz-bin/qcat?]/A+A/570/A64
}

infer atmospheric properties (e.g. Winn 2011). The Wide Angle Search for Planets (WASP; Pollacco et al. 2006) and other widefield ground-based surveys such as HAT-net (Bakos et al. 2002) are the leading discoverers of bright $(8.5 \lesssim V \lesssim 12.5)$ transiting systems, with around 150 planets between them.

Only a handful of the nearly one hundred planets discovered by WASP have orbital periods, $P$, greater than $5 \mathrm{~d}$. This is because there is an intrinsic pile-up of planets at three to five days, and hence a relative dearth of planets at longer periods. This is further exacerbated by the fact that at long periods the probability that a planetary system is aligned such that transits are visible from Earth is significantly reduced. Furthermore, because a large number of transits must be observed in order to overcome the correlated noise present in wide-field survey photometry (e.g. Smith et al. 2006), very long observational baselines are usually required to find longer period systems.

Recently, Lendl et al. (2014) reported the discovery of WASP-117b, the first WASP discovery with $P>9 \mathrm{~d}$. Only two other systems with $P>9$ d have been discovered from the ground by means of transits: HAT-P-15b (Kovács et al. 2010) 
and HAT-P-17b (Howard et al. 2012). Here we report the discovery of the fourth such planet, WASP-106b $(P=9.29 \mathrm{~d})$, along with a much shorter period planet, WASP-104b $(P=1.75 \mathrm{~d})$. Both planets orbit $V=11$ stars lying in the constellation Leo.

\section{Observations}

\subsection{WASP photometry}

Both WASP-104 and WASP-106 were observed for four seasons by SuperWASP-N at the Observatorio del Roque de los Muchachos on La Palma, Spain, from 2008 February 05 to 2011 March 29. Each of the targets was also observed for two seasons by WASP-South at the South African Astronomical Observatory near Sutherland, South Africa, from 2009 January 16 to 2010 May 30. These observations resulted in 24632 data points on WASP-104 (13993 from SuperWASP-N and 10639 from WASP-South) and 23624 measurements of WASP-106 (13101 from SuperWASP-N and 10523 from WASP-South).

The WASP instruments consist of eight Canon $200 \mathrm{~mm}$ f/1.8 lenses, each equipped with an Andor 2048×2048 e2v CCD camera, on a single robotic mount. Further details of the instrument, survey, and data reduction procedures are described in Pollacco et al. (2006), and details of the candidate selection procedure can be found in Collier Cameron et al. (2007) and Pollacco et al. (2008).

The data revealed the presence of transit-like signals with a period of around 1.75 days in the case of WASP-104 and around 9.29 days for WASP-106. The combined WASP light curves are shown binned and folded on the best-fitting orbital periods in the upper panels of Figs. 1 and 2. The light curves were also searched for additional transiting components with periods up to $120 \mathrm{~d}$, using the method described in Smith et al. (2009), but no such signals were found.

\subsection{Spectroscopic follow-up}

Spectroscopic observations of both targets were conducted using the SOPHIE spectrograph mounted on the $1.93-\mathrm{m}$ telescope of the Observatoire de Haute-Provence, France, and with CORALIE on the 1.2-m Euler-Swiss telescope at La Silla, Chile. A total of 21 observations (10 with CORALIE, 11 with SOPHIE) were made of WASP-104 between 2013 January 08 and 2013 June 25. WASP-106 was observed 29 times (20 times with CORALIE, 9 with SOPHIE) between 2013 January 06 and 2014 February 08.

SOPHIE was used in high-efficiency $(R=40000)$ mode, with the CCD in slow-readout mode. A second fibre was placed on the sky to check for sky background contamination in the spectra of the target. Corrections of up to a few tens of $\mathrm{m} \mathrm{s}^{-1}$ were applied to some exposures contaminated by moonlight. More information about the SOPHIE instrument can be found in Bouchy et al. (2009). The CORALIE observations were conducted only in dark time to avoid moon light entering the fibre.

Observations of thorium-argon emission line lamps were used to calibrate both the SOPHIE and CORALIE spectra. The data were processed using the standard SOPHIE and CORALIE data-reduction pipelines. The resulting RV data are listed in Table 1 and plotted in the lower panels of Figs. 1 and 2. In order to rule out the possibility that either system is a blended eclipsing binary, or that the RV variation is a result of stellar activity, we examined the bisector spans (e.g. Queloz et al. 2001). For
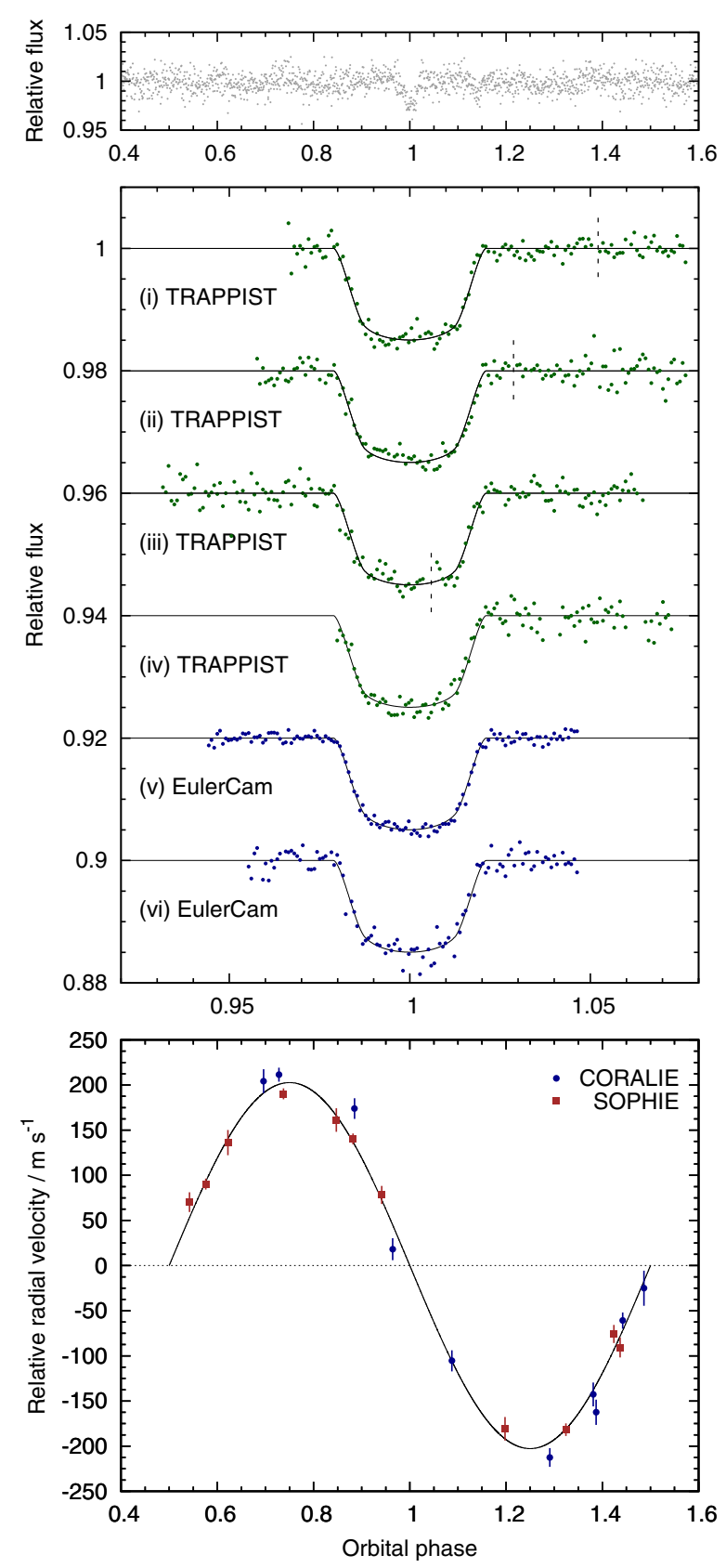

Fig. 1. WASP-104 photometry and radial velocities. Upper panel: phase-folded photometry from SuperWASP-N and WASP-South. Data are binned in phase with a bin-width equal to 120 s. Middle panel: high-precision follow-up photometry. Each light curve is offset in flux for clarity, and binned using a $120 \mathrm{~s}$ bin width. The light curves are identified with the same numbers as those in Table 2. Our best-fitting model is plotted with a solid line and TRAPPIST's meridian flips are marked with dashed vertical lines. Lower panel: RV measurements from CORALIE and SOPHIE, along with our best-fitting MCMC solution (solid line). The jitter added to the RV uncertainties is not shown. The centre-of-mass velocity, $\gamma=28.548042 \mathrm{~km} \mathrm{~s}^{-1}$, has been subtracted, as has the fitted offset $\gamma_{\mathrm{COR}-\mathrm{SOPH}}=280.23 \mathrm{~m} \mathrm{~s}^{-1}$ between the two datasets.

both stars, the bisector spans exhibit no significant correlation with RV (Fig. 3), as expected for true planetary systems.

\subsection{Photometric follow-up}

High-precision photometric follow-up observations were made from ESO's La Silla Observatory in Chile and from the 

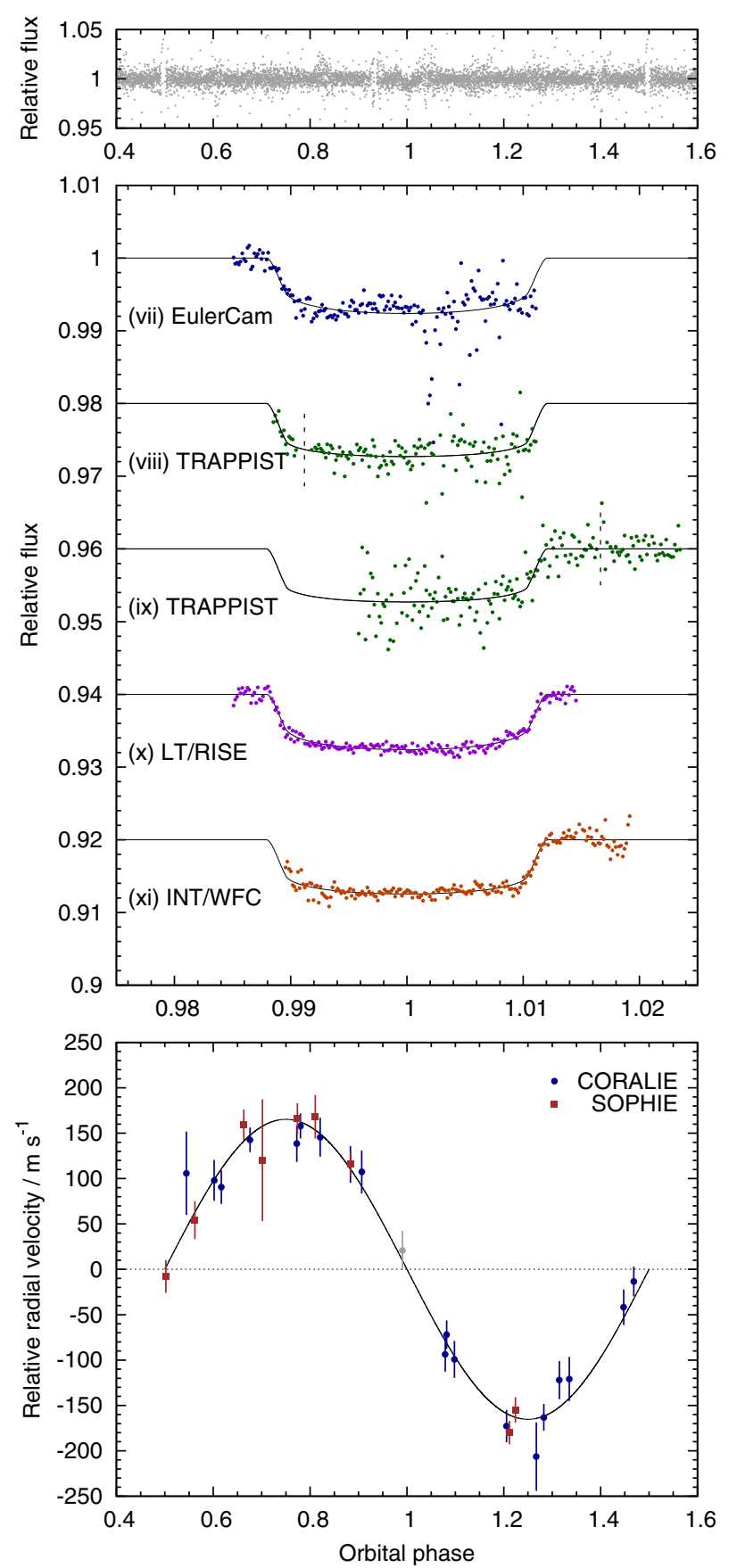

Fig. 2. WASP-106 photometry and radial velocities. Upper panel: phase-folded photometry from SuperWASP-N and WASP-South. Data are binned in phase with a bin-width equal to $120 \mathrm{~s}$. Middle panel: high-precision follow-up photometry. Each light curve is offset in flux for clarity, and binned using a $120 \mathrm{~s}$ bin width. The light curves are identified with the same numbers as those in Table 2. Our best-fitting model is plotted with a solid line and TRAPPIST's meridian flips are marked with dashed vertical lines. Lower panel: RV measurements from CORALIE and SOPHIE, along with our best-fitting MCMC solution (solid line). The CORALIE point taken during transit and excluded from our MCMC analysis is shown in grey. The centre-of-mass velocity, $\gamma=17.24744 \mathrm{~km} \mathrm{~s}^{-1}$, has been subtracted, as has the fitted offset $\gamma_{\mathrm{COR}-\mathrm{SOPH}}=-59.57 \mathrm{~m} \mathrm{~s}^{-1}$ between the two datasets.

Observatorio del Roque de los Muchachos on La Palma, Spain. The telescopes used were the robotic Transiting Planets and Planetesimals Small Telescope (TRAPPIST; Jehin et al. 2011) and the Euler-Swiss telescope with EulerCam

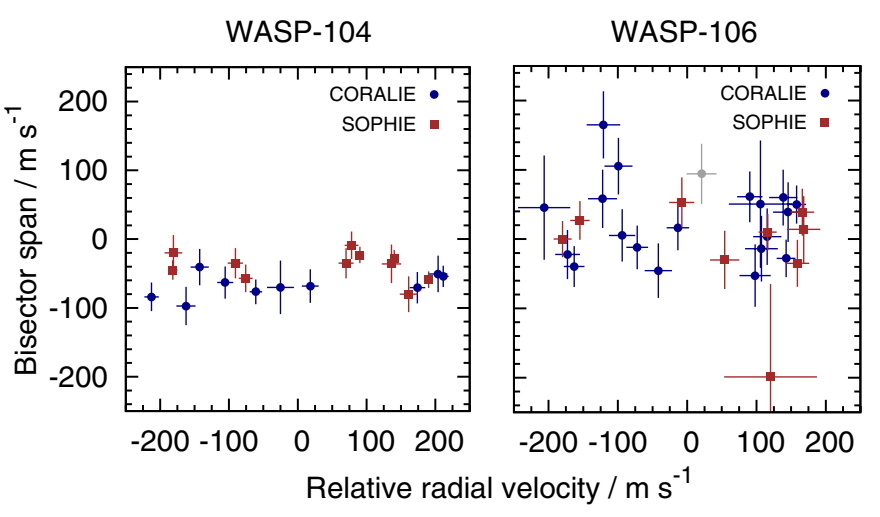

Fig. 3. Radial velocity bisector span vs. relative radial velocity for WASP-104 (left) and WASP-106 (right). The indicated uncertainties in the RVs do not include the added jitter, and the uncertainties in the bisector span are taken to be twice the uncertainty in the radial velocity. The CORALIE point taken during transit and excluded from our MCMC analysis is shown in grey.

Table 2. Observing log for follow-up transit photometry.

\begin{tabular}{|c|c|c|c|c|}
\hline No. & Date/UT & Telescope & Band & Full? ${ }^{\dagger}$ \\
\hline \multicolumn{5}{|c|}{ WASP-104: } \\
\hline (i) & 2013 Mar. 29/30 & TRAPPIST & $I+z$ & $\mathrm{Y}$ \\
\hline (ii) & 2013 Apr. 05/06 & TRAPPIST & $I+z$ & $\mathrm{Y}$ \\
\hline (iii) & 2013 Apr. $12 / 13$ & TRAPPIST & $I+z$ & $\mathrm{Y}$ \\
\hline (iv) & 2013 Jun. 02/03 & TRAPPIST & $I+z$ & $\mathrm{~N}$ \\
\hline (v) & 2013 Apr. 20 & Euler & I & $\mathrm{Y}$ \\
\hline (vi) & 2013 Apr. 27 & Euler & $I$ & $\mathrm{Y}$ \\
\hline \multicolumn{5}{|c|}{ WASP-106: } \\
\hline (vii) & 2013 May $13 / 14$ & Euler & Gunn $r$ & $\mathrm{~N}$ \\
\hline (viii) & 2013 May $13 / 14$ & TRAPPIST & $I+z$ & $\mathrm{~N}$ \\
\hline (ix) & 2014 Feb. $25 / 26$ & TRAPPIST & $I+z$ & $\mathrm{~N}$ \\
\hline (x) & 2014 Feb. $25 / 26$ & Liverpool & $V+R$ & Y \\
\hline (xi) & 2014 Feb. 25/26 & Isaac Newton & $i^{\prime}$ & $\mathrm{N}$ \\
\hline
\end{tabular}

Notes. ${ }^{(\dagger)}$ Indicates whether or not the complete transit was observed.

(Lendl et al. 2012) at La Silla; and the Liverpool Telescope (LT) with the Rapid Imaging Search for Exoplanets camera (RISE; Steele et al. 2008; Gibson et al. 2008) and the Isaac Newton Telescope (INT) with the Wide Field Camera (WFC) on La Palma. A summary of these observations is given in Table 2. Due to the relatively long transit duration of over five hours, it proved difficult to observe a full transit of WASP-106b. This was, however, achieved using the LT on the night of 2014 February 25/26. Simultaneous observations from the nearby INT were stymied by dome-shutter problems during ingress, preventing observation of the full transit, although a partial transit was observed.

\section{Determination of system parameters}

\subsection{Stellar parameters}

The individual CORALIE spectra were co-added to produce a single spectrum of each star with an average signal-to-noise ratio of around 60:1 for WASP-104 and 100:1 for WASP-106. The analyses were performed using standard pipeline reduction products and the procedures given in Doyle et al. (2013). The projected stellar rotation velocities, $v \sin i_{*}$, were determined by fitting the profiles of several unblended Fe I lines. We assumed macroturbulent velocities $\left(v_{\text {mac }}\right)$ of $2.5 \pm 0.6 \mathrm{~km} \mathrm{~s}^{-1}$ 
Table 3. Stellar parameters.

\begin{tabular}{lcc}
\hline \hline Parameter & WASP-104 & WASP-106 \\
\hline RA $(\mathrm{J} 2000.0)$ & $10^{\mathrm{h}} 42^{\mathrm{m}} 24.61^{\mathrm{s}}$ & $11^{\mathrm{h}} 05^{\mathrm{m}} 43.13^{\mathrm{s}}$ \\
Dec $(\mathrm{J} 2000.0)$ & $+07^{\circ} 26^{\prime} 06.3^{\prime \prime}$ & $-05^{\circ} 04^{\prime} 45.9^{\prime \prime}$ \\
Identifier & USNO-B1.0 $0974-0234922$ & $\mathrm{TYC} 4927-1063-1$ \\
$T_{* \text {,eff }} / \mathrm{K}$ & $5450 \pm 130$ & $6000 \pm 150$ \\
$\log g(\mathrm{cgs})$ & $4.5 \pm 0.2$ & $4.0 \pm 0.2$ \\
$\xi_{\mathrm{f}} / \mathrm{km} \mathrm{s}^{-1}$ & $0.9 \pm 0.1$ & $1.2 \pm 0.1$ \\
$v_{\mathrm{mac}} / \mathrm{km} \mathrm{s}^{-1}$ & $2.5 \pm 0.6$ & $4.7 \pm 0.6$ \\
$v \sin i_{*} / \mathrm{km} \mathrm{s}^{-1}$ & $0.4 \pm 0.7$ & $6.3 \pm 0.7$ \\
$\log A(\mathrm{Li})$ & $<0.7$ & $2.45 \pm 0.12$ \\
$\mathrm{Sp}$. Type & $\mathrm{G} 8$ & $\mathrm{~F} 9$ \\
Distance $/ \mathrm{pc}$ & $143 \pm 10$ & $283 \pm 21$ \\
$\mathrm{Mass} / M_{\odot}$ & $1.02 \pm 0.09$ & $1.27 \pm 0.15$ \\
$\mathrm{Radius} / R_{\odot}$ & $0.93 \pm 0.23$ & $1.85 \pm 0.52$ \\
{$[\mathrm{Fe} / \mathrm{H}]$} & $+0.32 \pm 0.09$ & $-0.09 \pm 0.09$ \\
$V$-mag $(\mathrm{Tycho})$ & $11.12 \pm 0.10$ & $11.21 \pm 0.11$ \\
\hline
\end{tabular}

Notes. The spectral type was estimated from $T_{*, \text { eff }}$ using the table of (Gray 2008, p. 507). The mass and radius were estimated using the Torres et al. (2010) calibration.

for WASP-104 and $4.7 \pm 0.6 \mathrm{~km} \mathrm{~s}^{-1}$ for WASP-106 using the asteroseismic-based calibration of Doyle et al. (2014) and an instrumental resolution of 55000 . The resultant stellar parameters are given in Table 3 .

\subsubsection{Stellar activity}

We searched the WASP photometry for rotational modulations, using the sine-wave fitting algorithm described by Maxted et al. (2011). We estimated the significance of periodicities by subtracting the fitted transit light curve and then repeatedly and randomly permuting the nights of observation. No significant periodic modulation was detected, to a 95 per cent confidence upper limit of 4 mmag in the case of WASP-104, and 1 mmag for WASP-106.

\subsubsection{Stellar age}

The low lithium abundance for WASP-104 suggests that the star is old (several Gyr, perhaps more than 5 Gyr; Sestito \& Randich 2005), and the low $v \sin i_{*}$ is consistent with this. From an analysis (Fig. 5) performed using the isochrones of Bressan et al. (2012), we infer an age of around $3 \pm 2$ Gyr.

The lithium abundance of WASP-106 suggests an age of around 1-2 Gyr. The $v \sin i_{*}$ gives an upper limit to the age of $1.4_{-0.4}^{+0.7}$ Gyr from the gyrochronological relation of Barnes (2007). Our isochrone analysis (Fig. 6) gives an age estimate of $7 \pm 2$ Gyr.

Such discrepancies in the ages estimated from these two methods are not unusual, particularly for estimates derived from isochrone fitting (e.g. Brown 2014).

\subsubsection{Stellar distance}

We calculated the distances to WASP-104 and WASP-106 (see Table 3) using Tycho apparent $V$-band magnitudes of $11.12 \pm$ 0.10 and $11.21 \pm 0.11$ respectively. The stellar luminosities were calculated from our best-fitting stellar effective temperatures and radii (Table 4), and we adopted bolometric corrections (from Flower 1996) of -0.15 and -0.05 , for WASP-104 and WASP-106 respectively. We use the interstellar Na D lines

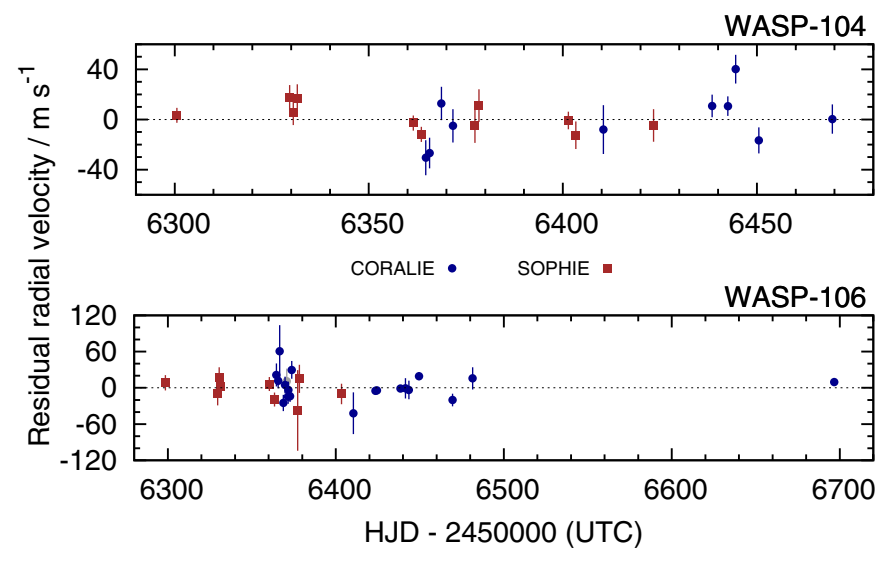

Fig. 4. Residual radial velocity as a function of time for WASP-104 (upper) and WASP-106 (lower). There is no evidence of radial acceleration in either system. The indicated uncertainties in the RVs do not include the added jitter. The CORALIE point taken during transit and excluded from our MCMC analysis is shown in grey.

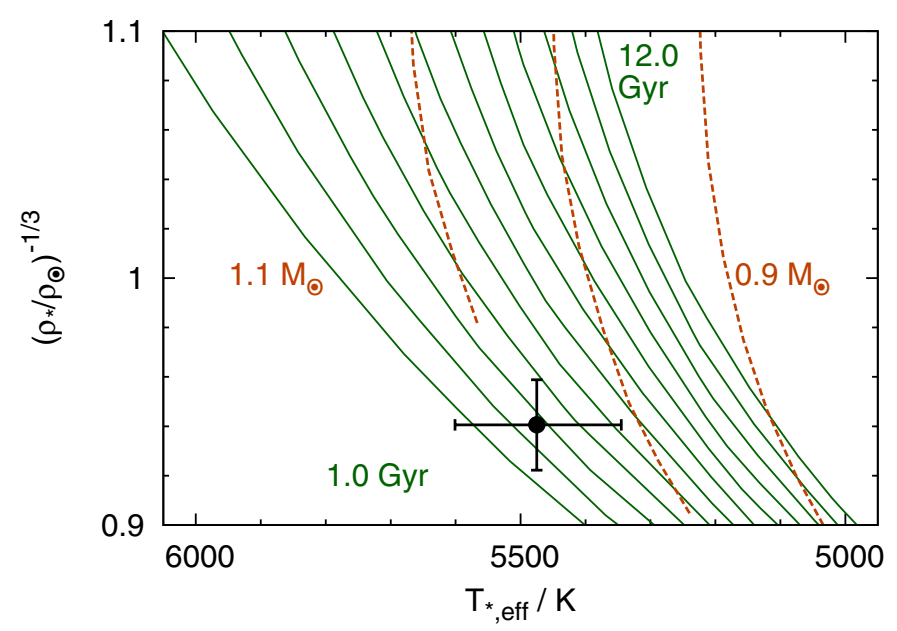

Fig. 5. Modified Hertzsprung-Russell diagram for WASP-104 (black circle). Isochrones (solid green lines) from the models of Bressan et al. (2012) are plotted for ages between 1.0 and $12.0 \mathrm{Gyr}$, spaced at $1.0 \mathrm{Gyr}$ intervals. Also shown are evolutionary tracks (dashed orange lines) for 0.9, 1.0 and $1.1 M_{\odot}$ from Bertelli et al. (2008).

and the calibration of Munari \& Zwitter (1997) to adopt colour excesses, $E(B-V)=0.04 \pm 0.01$ and $E(B-V)=0.02 \pm$ 0.01 for WASP-104 and WASP-106 respectively, to correct for interstellar reddening.

\subsection{Planetary system parameters}

For each system the photometric data were combined with the RVs and analysed simultaneously using the Markov chain Monte Carlo (MCMC) method.

We use the current version of the MCMC code described in Collier Cameron et al. (2007) and Pollacco et al. (2008), which uses the relation between stellar mass, effective temperature, density and metallicity appropriate for stars with masses between 0.2 and $3.0 M_{\odot}$ from Southworth (2011). Briefly, the $\mathrm{RV}$ data are modelled with a Keplerian orbit and the photometric transits are fitted using the model of Mandel \& Agol (2002) and limb-darkening was accounted for using a four-coefficient, non-linear model, employing coefficients appropriate to the passbands from the tabulations of Claret $(2000,2004)$. The 


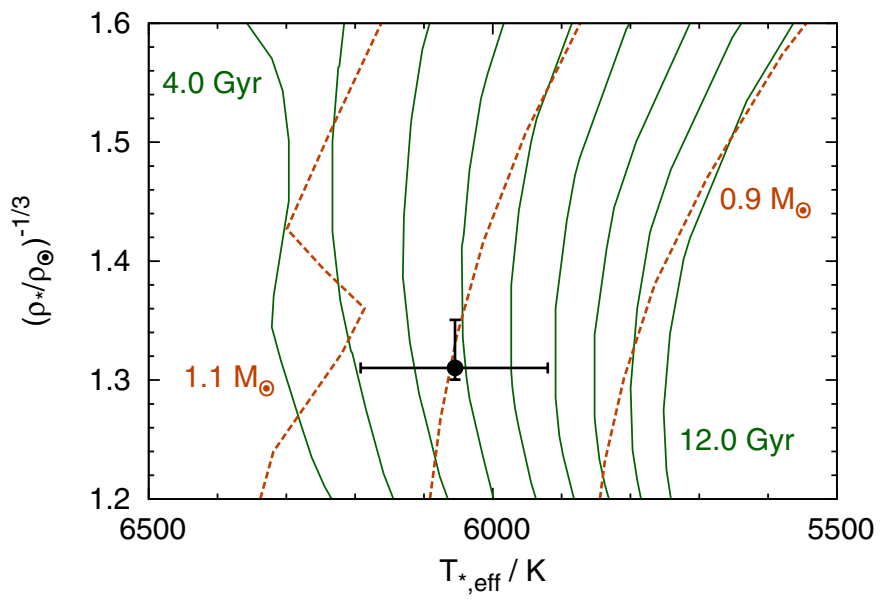

Fig. 6. Modified Hertzsprung-Russell diagram for WASP-106 (black circle). Isochrones (solid green lines) from the models of Bressan et al. (2012) are plotted for ages between 4.0 and $12.0 \mathrm{Gyr}$, spaced at $1.0 \mathrm{Gyr}$ intervals. Also shown are evolutionary tracks (dashed orange lines) for 0.9, 1.0 and 1.1 $M_{\odot}$ from Bertelli et al. (2008).

coefficients were determined using an initial interpolation in $\log g_{*}$ and $[\mathrm{Fe} / \mathrm{H}]$ (values from Table 3 ), and an interpolation in $T_{*, \text { eff }}$ at each MCMC step. The coefficient values corresponding to the best-fitting value of $T_{*, \text { eff }}$ are given in Table 5 .

The MCMC proposal parameters we use are: the epoch of mid-transit, $T_{\mathrm{c}}$; the orbital period, $P$; the transit duration, $T_{14}$; the fractional flux deficit that would be observed during transit in the absence of stellar limb-darkening, $\Delta F$; the transit impact parameter for a circular orbit, $b=a \cos \left(i_{P}\right) / R_{*}$; the stellar reflex velocity semi-amplitude, $K_{*}$; the stellar effective temperature, $T_{* \text {,eff }}$, and the stellar metallicity, $[\mathrm{Fe} / \mathrm{H}]$. When fitting for a non-zero orbital eccentricity, there are an additional two proposal parameters: $\sqrt{e} \cos \omega$ and $\sqrt{e} \sin \omega$, where $e$ is the orbital eccentricity, and $\omega$ is the argument of periastron (Anderson et al. 2011). The best-fitting system parameters are taken to be the median values of the posterior probability distribution, and are recorded in Table 4.

\subsubsection{WASP-104}

There is a star of approximately equal brightness to WASP-104 (USNO-B1.0 $R=10.82$, cf. 11.28 for WASP-104), located 29" to the north. We found from our observations with the Euler Telescope that this neighbouring object (TYC 260-1073-1) is itself a visual binary, with the components separated from each other by around $1^{\prime \prime}$. Further, we found this binary to be unrelated to WASP-104, which has a systemic RV of $28 \mathrm{~km} \mathrm{~s}^{-1}$ compared to $-3 \mathrm{~km} \mathrm{~s}^{-1}$ for the binary. This conclusion is supported by proper motions from the UCAC4 catalogue (Zacharias et al. 2012), which indicate that it is unlikely that WASP-104 $\left(\mu_{\alpha}=-30.3 \pm 2.7 \mathrm{mas} \mathrm{yr}^{-1} ; \mu_{\delta}=-2.3 \pm 1.6 \mathrm{mas} \mathrm{yr}^{-1}\right)$ and the neighbouring object $\left(\mu_{\alpha}=-14.4 \pm 6.6 \mathrm{mas} \mathrm{yr}^{-1} ; \mu_{\delta}=\right.$

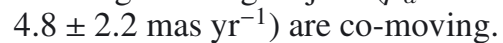

Although this object is easily excluded from the photometric apertures used in our follow-up observations, it falls within the 3.5-pixel (=48") WASP aperture, thus rendering the WASP photometry unreliable for measuring the transit depth. After determining the best-fitting orbital period from an MCMC analysis of all the available data, we exclude the WASP photometry from subsequent analysis. Instead, we fix the orbital period to the value previously determined and use MCMC to analyse only the follow-up photometry and the RVs.

An initial fit for an eccentric orbit gave $e=0.0092^{+0.0123}$ (with $\omega=15_{-125}^{+106}$ degrees). This is consistent with a circular orbit; according to the F-test of Lucy \& Sweeney (1971) there is a $>99$ percent chance that the apparent eccentricity could have arisen if the underlying orbit were circular. We also tried fitting for a drift in the RVs by fitting for a linear function in time. Such a trend may be indicative of a third body in the system, but fitting for radial acceleration, $\mathrm{d} \gamma / \mathrm{d} t$, is clearly not justified in this case, because we obtain $\mathrm{d} \gamma / \mathrm{d} t=-24 \pm 28 \mathrm{~m} \mathrm{~s}^{-1} \mathrm{yr}^{-1}$ (Fig. 4).

Linear functions of time were fitted to each light curve at each step of the MCMC, to remove systematic trends. We added "jitter" terms of $15 \mathrm{~m} \mathrm{~s}^{-1}$ for CORALIE and $6 \mathrm{~m} \mathrm{~s}^{-1}$ for SOPHIE to the formal RV uncertainties in quadrature so as to obtain a spectroscopic reduced $\chi^{2}$ of unity.

\subsubsection{WASP -106}

One of the CORALIE RV measurements was made during transit, and we exclude this point from our MCMC analysis because we do not model the Rossiter-McLaughlin effect. The excluded point is, however, plotted (in grey) in the lower panel of Fig. 2. It was not necessary to add any "jitter" to the RV uncertainties.

Fitting for an eccentric orbit gives $e=0.031_{-0.022}^{+0.032}$ (with $\omega=103_{-82}^{+30}$ degrees). This is again consistent with a circular orbit (the Lucy \& Sweeney 1971 F-test probability is 12 per cent). The best-fitting radial acceleration is $\mathrm{d} \gamma / \mathrm{d} t=11_{-18}^{+13} \mathrm{~m} \mathrm{~s}^{-1} \mathrm{yr}^{-1}$, which is also consistent with zero (Fig. 4). We therefore adopt, as for the WASP-104 system, a circular orbit and a constant systemic RV.

Linear functions of time were fitted to each light curve at each step of the MCMC, to remove systematic trends. In the case of the LT light curve (x), the function fitted was quartic, rather than linear in time. A function of CCD position, airmass and sky background instead of time was fitted to the INT light curve (xi). In each case, the addition of extra terms was justified by an improved value of the Bayesian Information Criterion (BIC; Schwarz 1978).

\section{Results and discussion}

We find that WASP-104 and WASP-106 are both main-sequence stars. WASP-104 has a mass and radius similar to that of the Sun, while WASP-106 is around 20 per cent more massive than the Sun, and has a radius around 40 per cent larger. Both planets are more massive than Jupiter (WASP-104b has a mass of $1.27 \pm$ $0.05 M_{\text {Jup }}$, while WASP-106b has a mass of $1.93 \pm 0.08 M_{\text {Jup }}$ ). WASP-104b and WASP-106b are not bloated like many hot Jupiters. Indeed with radii of $1.14 \pm 0.04$ and $1.09 \pm 0.04 R_{\text {Jup }}$ respectively, neither planet is much larger than Jupiter.

\subsection{The circular nature of the orbit of WASP-106b}

It is interesting to compare WASP-106 to WASP-117 (Lendl et al. 2014), a recently discovered transiting system consisting of a hot Jupiter in a $10.0 \mathrm{~d}$ orbit that is both significantly eccentric $(e=0.302 \pm 0.023)$ and misaligned $\left(\psi=69.6_{-4.1}^{+4.7} \mathrm{deg}\right.$; $\beta=-44 \pm 11 \mathrm{deg}$ ). By contrast, WASP-106b orbits in a circular or near-circular orbit (Sect. 3.2.2). The stars WASP-106 and WASP-117 $\left(M_{*}=1.126 \pm 0.029 M_{\odot}, R_{*}=1.170_{-0.059}^{+0.067} R_{\odot}\right.$, $T_{*, \text { eff }}=6038 \pm 88 \mathrm{~K}$ ) are also very similar to each other. 
Table 4. System parameters for WASP-104 and WASP-106.

\begin{tabular}{|c|c|c|c|c|}
\hline Parameter & Symbol & Unit & WASP-104 & WASP-106 \\
\hline \multicolumn{5}{|l|}{ Model parameters: } \\
\hline Orbital period & $P$ & $\mathrm{~d}$ & $1.7554137_{-0.0000036}^{+0.0000018} \dagger$ & $9.289715 \pm 0.000010$ \\
\hline Epoch of mid-transit & $T_{\mathrm{c}}$ & HJD, UTC & $2456406.11126 \pm 0.00012$ & $2456649.54972 \pm 0.00033$ \\
\hline Transit duration & $T_{14}$ & d & $0.07342 \pm 0.00056$ & $0.22346_{-0.00090}^{+0.00104}$ \\
\hline Planet-to-star area ratio & $\Delta F=R_{\mathrm{p}}^{2} / R_{*}^{2}$ & $\ldots$ & $0.01474 \pm 0.00020$ & $0.00642 \pm 0.00018$ \\
\hline Transit impact parameter & $b$ & $\ldots$ & $0.724 \pm 0.014$ & $0.127_{-0.087}^{+0.149}$ \\
\hline Stellar orbital velocity semi-amplitude & $K_{*}$ & $\mathrm{~m} \mathrm{~s}^{-1}$ & $202.7 \pm 4.3$ & $165.3 \pm 4.3$ \\
\hline System velocity & $\gamma$ & $\mathrm{km} \mathrm{s}^{-1}$ & $28.54804 \pm 0.00010$ & $17.24744 \pm 0.00019$ \\
\hline Velocity offset between CORALIE and SOPHIE & $\gamma_{\mathrm{COR}-\mathrm{SOPH}}$ & $\mathrm{m} \mathrm{s}^{-1}$ & $280.2 \pm 1.0$ & $-59.57 \pm 0.13$ \\
\hline Stellar effective temperature & $T_{*, \mathrm{eff}}$ & K & $5475 \pm 127$ & $6055 \pm 136$ \\
\hline Stellar metallicity & {$[\mathrm{Fe} / \mathrm{H}]$} & $\operatorname{dex}$ & $+0.320 \pm 0.090$ & $-0.090 \pm 0.090$ \\
\hline \multicolumn{5}{|l|}{ Derived parameters: } \\
\hline Ingress/egress duration & $T_{12}=T_{34}$ & $\mathrm{~d}$ & $0.01535 \pm 0.00075$ & $0.01685_{-0.00039}^{+0.00118}$ \\
\hline Orbital inclination angle & $i_{\mathrm{P}}$ & $\circ$ & $83.63 \pm 0.25$ & $89.49_{-0.64}^{-0.35}$ \\
\hline Orbital eccentricity (adopted) & $e$ & $\ldots$ & 0 & 0 \\
\hline Orbital eccentricity (3- $\sigma$ upper-limit) & $\ldots$ & $\ldots$ & 0.060 & 0.13 \\
\hline Stellar mass & $M_{*}$ & $M_{\odot}$ & $1.076 \pm 0.049$ & $1.192 \pm 0.054$ \\
\hline Stellar radius & $R_{*}$ & $R_{\odot}$ & $0.963 \pm 0.027$ & $1.393_{-0.028}^{+0.048}$ \\
\hline $\log$ (stellar surface gravity) & $\log g_{*}$ & (cgs) & $4.503 \pm 0.016$ & $4.226_{-0.022}^{+0.010}$ \\
\hline Stellar density & $\rho_{*}$ & $\rho_{\odot}$ & $1.207 \pm 0.070$ & $0.445_{-0.039}^{+0.022}$ \\
\hline Planet mass & $M_{\mathrm{P}}$ & $M_{\text {Jup }}$ & $1.272 \pm 0.047$ & $1.925 \pm 0.076$ \\
\hline Planet radius & $R_{\mathrm{P}}$ & $R_{\text {Jup }}$ & $1.137 \pm 0.037$ & $1.085_{-0.028}^{+0.046}$ \\
\hline $\log$ (planet surface gravity) & $\log g_{\mathrm{P}}$ & (cgs) & $3.353 \pm 0.023$ & $3.571_{-0.031}^{+0.028}$ \\
\hline Planet density & $\rho_{\mathrm{P}}$ & $\rho_{\mathrm{J}}$ & $0.866 \pm 0.071$ & $1.50_{-0.16}^{+0.11}$ \\
\hline Scaled orbital major semi-axis & $a / R_{*}$ & $\ldots$ & $6.52 \pm 0.13$ & $14.20_{-0.43}^{+0.11}$ \\
\hline Orbital major semi-axis & $a$ & $\mathrm{au}$ & $0.02918 \pm 0.00045$ & $0.0917 \pm 0.0014$ \\
\hline $\begin{array}{l}\text { Planet equilibrium temperature } \\
\text { (uniform heat redistribution) }\end{array}$ & $T_{\mathrm{P}, \mathrm{A}=0}$ & $\mathrm{~K}$ & $1516 \pm 39$ & $1140 \pm 29$ \\
\hline
\end{tabular}

Notes. ${ }^{(\dagger)}$ The orbital period of WASP-104b and its uncertainty was determined from a fit to all the data (including photometry from the WASP instruments). The period was fixed in subsequent analyses (which excluded the WASP photometry). See Sect. 3.2.1. The following constant values are used: $\mathrm{au}=1.49598 \times 10^{11} \mathrm{~m} ; R_{\odot}=6.9599 \times 10^{8} \mathrm{~m} ; M_{\odot}=1.9892 \times 10^{30} \mathrm{~kg} ; R_{\text {Jup }}=7.1492 \times 10^{7} \mathrm{~m} ; M_{\text {Jup }}=1.89896 \times 10^{27} \mathrm{~kg}$; $\rho_{\mathrm{J}}=1240.67 \mathrm{~kg} \mathrm{~m}^{-3}$.

Table 5. Stellar limb-darkening coefficients.

\begin{tabular}{lcccrrrr}
\hline \hline Star & Instruments & Observation bands & Claret band & $a_{1}$ & $a_{2}$ & $a_{3}$ & $a_{4}$ \\
\hline WASP-104 & TRAPPIST & Cousins $I+$ Sloan $z^{\prime}$ & Sloan $z^{\prime}$ & 0.821 & -0.828 & 1.212 & -0.527 \\
WASP-104 & Euler & Cousins $I$ & Cousins $I$ & 0.821 & -0.835 & 1.308 & -0.573 \\
WASP-106 & WASP/Euler $/$ LT & Broad $($ 400-700 nm)/Gunn $r / V+R$ & Cousins $R$ & 0.497 & 0.273 & 0.034 & -0.096 \\
WASP-106 & TRAPPIST & Cousins $I+$ Sloan $z^{\prime}$ & Sloan $z^{\prime}$ & 0.592 & -0.134 & 0.337 & -0.199 \\
WASP-106 & INT & Sloan $i^{\prime}$ & Sloan $i^{\prime}$ & 0.559 & 0.033 & 0.223 & -0.162 \\
\hline
\end{tabular}

Such planets may offer important evidence as to which of two proposed planetary migration scenarios is dominant. Diskdriven migration should produce giant planets in circular orbits with low obliquities, whereas planet-planet scattering and migration through the Kozai mechanism should lead to highly eccentric orbits, with large obliquities (see Lendl et al. 2014 and references therein). These eccentric orbits may be subsequently circularised through tidal interactions with the star.

The vast majority of known hot Jupiters lie in circular or near-circular orbits, and distinguishing between established circular orbits and once-eccentric orbits that have been relatively quickly made circular by the huge tidal forces at play is non-trivial. Hot Jupiters with periods longer than $5 \mathrm{~d}$, where the observed pile-up lies, may offer a clue to this puzzle, because the tidal forces acting to circularise the orbit are lesser.

Using Eq. (1) of Jackson et al. (2008), we calculate the circularisation time-scale, $\tau_{\mathrm{e}}=\left(\frac{1}{e} \frac{\mathrm{d} e}{\mathrm{~d} t}\right)^{-1}$, for the orbit of WASP-106b, in terms of the tidal dissipation parameters for the planet, $Q_{\mathrm{P}}$, and for the star, $Q_{*}$,

$\tau_{\mathrm{e}}=\left(\frac{0.046}{\left(\frac{Q_{\mathrm{P}}}{10^{5.5}}\right)}+\frac{0.0023}{\left(\frac{Q_{*}}{10^{6.5}}\right)}\right)^{-1} \mathrm{Gyr}$ 
Adopting $Q_{\mathrm{P}}=10^{5.5}$ and $Q_{*}=10^{6.5}$ (the best-fitting values from the study of Jackson et al. 2008), we find $\tau_{\mathrm{e}}=20.7 \mathrm{Gyr}^{1}$. Even in the extreme case that $Q_{\mathrm{P}}=Q_{*}=10^{5}$, the circularisation timescale is still as long as $4.6 \mathrm{Gyr}$, comparable to the age of the system. Hence it seems probable that the orbit of WASP-106b has not been circularised from a highly eccentric starting point, but rather that the orbit has remained close to circular for the lifetime of the system.

A large fraction of similar planets, however, lie on significantly eccentric orbits. Using the Exoplanets Data Explorer (Wright et al. 2011), we find that there are 34 known planets of at least half the mass of Jupiter with orbital periods in the range $9 \leq P \leq 100 \mathrm{~d}$ (including WASP-106b). Around half of these (18) are known to have significantly eccentric $(e \geq 0.1)$ orbits, while the remainder have near-circular orbits, or their eccentricity is unknown.

It would be interesting to see if the obliquity of the WASP-106 system is also low, as well as the eccentricity. Measuring the sky-projected angle between the stellar spin axis and the orbital axis through the Rossiter-McLaughlin effect would give an insight into this. Such a measurement should prove possible; we predict an amplitude of around $26 \mathrm{~m} \mathrm{~s}^{-1}$ for the Rossiter-McLaughlin effect, and a typical RV precision of $4 \mathrm{~m} \mathrm{~s}^{-1}$ per $10 \mathrm{~min}$ exposure with HARPS.

Acknowledgements. We acknowledge the significant contributions made to the construction and operation of WASP-South by Coel Hellier, who also identified WASP-104 and WASP-106 as candidate planetary systems. WASP-South is hosted by the SAAO and SuperWASP by the Isaac Newton Group and the Instituto de Astrofísica de Canarias; we gratefully acknowledge their ongoing support and assistance. Funding for WASP comes from consortium universities and from the UK's Science and Technology Facilities Council (STFC). TRAPPIST is a project funded by the Belgian Fund for Scientific Research (FNRS) with the participation of the Swiss National Science Foundation. M.G. and E.J. are FNRS Research Associates. The Liverpool Telescope is operated on the island of La Palma by Liverpool John Moores University in the Spanish Observatorio del Roque de los Muchachos of the Instituto de Astrofísica de Canarias with financial support from the UK STFC; observations were made under programme PL14A10. The Isaac Newton Telescope is operated on the island of La Palma by the Isaac Newton Group in the Spanish Observatorio del Roque de los Muchachos of the Instituto de Astrofísica de Canarias; observations were made under programme I/2014A/09. AHMJT is a Swiss National Science Foundation fellow under grant number P300P2-147773.

\section{References}

Anderson, D. R., Collier Cameron, A., Hellier, C., et al. 2011, ApJ, 726, L19 Bakos, G. Á., Lázár, J., Papp, I., Sári, P., \& Green, E. M. 2002, PASP, 114, 974 Barnes, S. A. 2007, ApJ, 669, 1167

Bertelli, G., Girardi, L., Marigo, P., \& Nasi, E. 2008, A\&A, 484, 815

Bouchy, F., Hébrard, G., Udry, S., et al. 2009, A\&A, 505, 853

Bressan, A., Marigo, P., Girardi, L., et al. 2012, MNRAS, 427, 127

Brown, D. J. A. 2014, MNRAS, 442, 1844

Claret, A. 2000, A\&A, 363, 1081

Claret, A. 2004, A\&A, 428, 1001

Collier Cameron, A., Wilson, D. M., West, R. G., et al. 2007, MNRAS, 380, 1230

Doyle, A. P., Smalley, B., Maxted, P. F. L., et al. 2013, MNRAS, 428, 3164

Doyle, A. P., Davies, G. R., Smalley, B., Chaplin, W. J., \& Elsworth, Y. 2014, MNRAS, 444, 3592

Flower, P. J. 1996, ApJ, 469, 355

Gibson, N. P., Pollacco, D., Simpson, E. K., et al. 2008, A\&A, 492, 603

Gray, D. F. 2008, The Observation and Analysis of Stellar Photospheres, 3rd edn. (Cambridge University Press)

Howard, A. W., Bakos, G. Á., Hartman, J., et al. 2012, ApJ, 749, 134

Jackson, B., Greenberg, R., \& Barnes, R. 2008, ApJ, 678, 1396

Jehin, E., Gillon, M., Queloz, D., et al. 2011, The Messenger, 145, 2

Kovács, G., Bakos, G. Á., Hartman, J. D., et al. 2010, ApJ, 724, 866

Lendl, M., Anderson, D. R., Collier-Cameron, A., et al. 2012, A\&A, 544, A72

Lendl, M., Triaud, A. H. M. J., Anderson, D. R., et al. 2014, A\&A, 568, A81

Lucy, L. B., \& Sweeney, M. A. 1971, AJ, 76, 544

Mandel, K., \& Agol, E. 2002, ApJ, 580, L171

Maxted, P. F. L., Anderson, D. R., Collier Cameron, A., et al. 2011, PASP, 123, 547

Munari, U., \& Zwitter, T. 1997, A\&A, 318, 269

Pollacco, D. L., Skillen, I., Cameron, A. C., et al. 2006, PASP, 118, 1407

Pollacco, D., Skillen, I., Collier Cameron, A., et al. 2008, MNRAS, 385, 1576

Queloz, D., Henry, G. W., Sivan, J. P., et al. 2001, A\&A, 379, 279

Schwarz, G. 1978, Ann. Stat., 6, 461

Sestito, P., \& Randich, S. 2005, A\&A, 442, 615

Smith, A. M. S., Collier Cameron, A., Christian, D. J., et al. 2006, MNRAS, 373, 1151

Smith, A. M. S., Hebb, L., Collier Cameron, A., et al. 2009, MNRAS, 398, 1827 Southworth, J. 2011, MNRAS, 417, 2166

Steele, I. A., Bates, S. D., Gibson, N., et al. 2008, in SPIE Conf. Ser., 7014

Torres, G., Andersen, J., \& Giménez, A. 2010, A\&ARv, 18, 67

Winn, J. N. 2011, Exoplanet Transits and Occultations, ed. S. Seager, 55

Wright, J. T., Fakhouri, O., Marcy, G. W., et al. 2011, PASP, 123, 412

Zacharias, N., Finch, C. T., Girard, T. M., et al. 2012, VizieR Online Data Catalog: I/322 
Table 1. Radial velocities of WASP-104 and WASP-106.

\begin{tabular}{|c|c|c|c|c|c|}
\hline Star & $\begin{array}{l}\text { BJD(UTC) } \\
-2450000\end{array}$ & $\begin{array}{c}R V \\
\mathrm{~km} \mathrm{~s}^{-1}\end{array}$ & $\begin{array}{c}\sigma_{R V} \\
\mathrm{~km} \mathrm{~s}^{-1}\end{array}$ & $\begin{array}{c}\mathrm{BS} \\
\mathrm{km} \mathrm{s}^{-1}\end{array}$ & Inst. $^{\dagger}$ \\
\hline WASP-104 & 6364.6606 & 28.386 & 0.014 & -0.082 & COR. \\
\hline WASP-104 & 6365.6744 & 28.566 & 0.012 & -0.053 & COR. \\
\hline WASP-104 & 6368.7145 & 28.752 & 0.013 & -0.030 & COR. \\
\hline WASP-104 & 6371.6720 & 28.405 & 0.013 & -0.027 & COR. \\
\hline WASP-104 & 6410.4762 & 28.523 & 0.019 & -0.057 & COR. \\
\hline WASP-104 & 6438.4853 & 28.487 & 0.009 & -0.062 & COR. \\
\hline WASP-104 & 6442.4977 & 28.760 & 0.008 & -0.040 & COR. \\
\hline WASP-104 & 6444.5289 & 28.722 & 0.011 & -0.055 & COR. \\
\hline WASP-104 & 6450.5074 & 28.336 & 0.010 & -0.069 & COR. \\
\hline WASP-104 & 6469.4597 & 28.443 & 0.012 & -0.048 & COR. \\
\hline WASP-104 & 6300.5794 & 28.968 & 0.006 & -0.028 & SOPH. \\
\hline WASP-104 & 6329.6172 & 28.752 & 0.010 & -0.057 & SOPH. \\
\hline WASP-104 & 6330.5262 & 28.906 & 0.010 & -0.009 & SOPH. \\
\hline WASP-104 & 6331.5802 & 28.898 & 0.011 & -0.035 & SOPH. \\
\hline WASP-104 & 6361.4814 & 28.918 & 0.006 & -0.023 & SOPH. \\
\hline WASP-104 & 6363.5205 & 29.018 & 0.006 & -0.059 & SOPH. \\
\hline WASP-104 & 6377.3614 & 28.964 & 0.014 & -0.036 & SOPH. \\
\hline WASP-104 & 6378.3721 & 28.647 & 0.013 & -0.020 & SOPH. \\
\hline WASP-104 & 6401.4142 & 28.646 & 0.007 & -0.045 & SOPH. \\
\hline WASP-104 & 6403.3674 & 28.737 & 0.011 & -0.035 & SOPH. \\
\hline WASP-104 & 6423.3975 & 28.989 & 0.013 & -0.080 & SOPH. \\
\hline WASP-106 & 6364.6843 & 17.127 & 0.024 & 0.165 & COR. \\
\hline WASP-106 & 6365.7257 & 17.206 & 0.020 & -0.045 & COR. \\
\hline WASP-106 & 6366.6264 & 17.353 & 0.046 & 0.051 & COR. \\
\hline WASP-106 & 6368.7463 & 17.386 & 0.020 & 0.060 & COR. \\
\hline WASP-106 & 6369.7726 & 17.363 & 0.020 & 0.004 & COR. \\
\hline WASP-106 & 6370.7735 & 17.268 & 0.022 & 0.094 & COR. \\
\hline WASP-106 & 6371.5952 & 17.154 & 0.019 & 0.005 & COR. \\
\hline WASP-106 & 6371.7690 & 17.148 & 0.020 & 0.105 & COR. \\
\hline WASP-106 & 6372.7689 & 17.075 & 0.018 & -0.022 & COR. \\
\hline WASP-106 & 6373.7840 & 17.125 & 0.021 & 0.058 & COR. \\
\hline WASP-106 & 6410.5005 & 17.041 & 0.038 & 0.045 & COR. \\
\hline WASP-106 & 6423.5873 & 17.390 & 0.014 & -0.028 & COR. \\
\hline WASP-106 & 6424.5533 & 17.405 & 0.014 & 0.050 & COR. \\
\hline WASP-106 & 6438.5133 & 17.084 & 0.015 & -0.040 & COR. \\
\hline WASP-106 & 6441.4779 & 17.345 & 0.023 & -0.053 & COR. \\
\hline WASP-106 & 6443.5126 & 17.393 & 0.021 & 0.039 & COR. \\
\hline WASP-106 & 6449.5284 & 17.234 & 0.016 & 0.017 & COR. \\
\hline WASP-106 & 6469.4842 & 17.338 & 0.018 & 0.061 & COR. \\
\hline WASP-106 & 6481.4663 & 17.355 & 0.024 & -0.014 & COR. \\
\hline WASP-106 & 6696.7609 & 17.175 & 0.016 & -0.012 & COR. \\
\hline WASP-106 & 6298.6199 & 17.033 & 0.014 & 0.027 & SOPH. \\
\hline WASP-106 & 6329.6333 & 17.242 & 0.021 & -0.030 & SOPH. \\
\hline WASP-106 & 6330.5696 & 17.347 & 0.017 & -0.035 & SOPH. \\
\hline WASP-106 & 6331.5970 & 17.354 & 0.017 & 0.039 & SOPH. \\
\hline WASP-106 & 6360.4915 & 17.304 & 0.013 & 0.010 & SOPH. \\
\hline WASP-106 & 6363.5345 & 17.008 & 0.013 & -0.000 & SOPH. \\
\hline WASP-106 & 6377.3763 & 17.308 & 0.067 & -0.199 & SOPH. \\
\hline WASP-106 & 6378.3889 & 17.356 & 0.024 & 0.014 & SOPH. \\
\hline WASP-106 & 6403.3926 & 17.180 & 0.018 & 0.053 & SOPH. \\
\hline
\end{tabular}

Notes. ${ }^{(\dagger)}$ Spectrograph used. COR. = CORALIE on the Euler-Swiss telescope; SOPH. = SOPHIE on the 1.93-m telescope at the OHP. 